\title{
Eighteen-month-olds selectively generalize words from accurate speakers to novel contexts
}

\author{
Elena Luchkina | David M. Sobel | James L. Morgan
}

Department of Cognitive, Linguistic and Psychological Sciences, Brown University, Providence, RI, USA

\section{Correspondence}

Elena Luchkina, Department of Cognitive, Linguistic and Psychological Sciences, Brown University, Providence, RI, USA.

Email: Elena_Luchkina@brown.edu

\section{Funding}

National Science Foundation grant (1223777) and National Institutes of Health grant (HD068501)

\begin{abstract}
The present studies examine whether and how 18-month-olds use informants' accuracy to acquire novel labels for novel objects and generalize them to a new context. In Experiment 1, two speakers made statements about the labels of familiar objects. One used accurate labels and the other used inaccurate labels. One of these speakers then introduced novel labels for two novel objects. At test, toddlers saw those two novel objects and heard an unfamiliar voice say one of the labels provided by the speaker. Only toddlers who had heard the novel labels introduced by the accurate speaker looked at the appropriate novel object above chance. Experiment 2 explored possible mechanisms underlying this difference in generalization. Rather than making statements about familiar objects' labels, both speakers asked questions about the objects' labels, with one speaker using accurate labels and the other using inaccurate labels. Toddlers' generalization of novel labels for novel objects was at chance for both speakers, suggesting that toddlers do not simply associate hearing the accurate label with the reliability of the speaker. We discuss these results in terms of potential mechanisms by which children learn and generalize novel labels across contexts from speaker reliability.
\end{abstract}

\section{RESEARCH HIGHLIGHTS}

- Toddlers generalize words learned from speakers who accurately label familiar objects, but not speakers who are inaccurate.

- When speakers ask questions that contain accurate or inaccurate labels for objects during familiarization, toddlers do not consider either speaker a reliable source of knowledge for novel labels.

- These data suggest that toddlers are using more than word-object-speaker associations to determine speaker reliability.

\section{1 | INTRODUCTION}

Because the relation between how a word sounds and what a word means is arbitrary and conventional (Saussure, 1916/1966), children must learn the meanings of words from other competent language users. Several studies have found that preschoolers use speakers' history of accuracy in labeling familiar words when they learn meanings of novel words. For example, Koenig and colleagues (e.g., Clément, Koenig, \& Harris, 2004; Koenig, Clément, \& Harris, 2004; Koenig \& Harris, 2005) have shown that 4-year-olds identified an informant who was accurate in labeling familiar objects as knowledgeable about the meaning of words, and predicted that she would be more likely to know the name of a novel object over an informant who was always inaccurate at labeling familiar objects.

Even before children reach preschool age, they exhibit an ability to track interlocutors' competence or accuracy and use it in learning situations. For example, Zmyj, Buttelmann, Carpenter, and Daum (2010) showed that 14-month-olds are less likely to imitate agents' novel actions when those agents use familiar objects incompetently (for example, putting a shoe on their hand instead of foot) than when agents use familiar objects competently. Similarly, 12-month-olds track informants' competence and use it in their own exploratory behavior (Stenberg, 2013). Even 8-month-old infants have the capacity to monitor the reliability of a potential informant's gaze and 
use it in planning their responses about whether interesting events will appear (Tummeltshammer, Wu, Sobel, \& Kirkham, 2014).

Despite converging evidence from investigations of infants' accuracy judgments in these non-verbal domains, studies examining younger children's learning of novel labels have reported conflicting evidence. Koenig and Woodward (2010) showed that 2-year-olds differentiated between speakers with different histories of accuracy when those speakers provided contrasting information. Brooker and Poulin-Dubois (2013) tested even younger children; they showed that 18-month-olds were more likely to learn words and actions from an informant who labeled familiar objects accurately than from a person who did so inaccurately. Krogh-Jespersen and Echols (2012), however, found that 2-year-olds accepted speakers' labels for novel objects regardless of whether those informants were previously accurate, inaccurate, or ignorant about labels for familiar objects.

In light of these conflicting findings, the present study had two primary goals. The first was to broaden the finding that 18-month-olds can use speakers' accuracy to make inferences about novel objects' labels. In particular, we were concerned with the generalization of word learning, in this case from a social context to a less social context with a novel, disembodied voice. A characteristic of most of the studies cited above is that a familiar individual asks for the target object using a novel label during test. ${ }^{1}$ While toddlers may form an association between the utterance, the speaker, and the object, it is not clear whether they acquire generalizable knowledge of object labels. In our work, we aimed to resolve two potential complications that result from using the same speaker during novel label training and test.

First, seeing the speaker who provided the novel labels might serve as contextual support (Hendrickson \& Sundara, 2017) and trigger object-label-speaker associations that toddlers may have formed during the training. Henderson, Graham, and Schell (2015) showed that 24-month-olds are open to learning novel labels from unreliable sources if they expect them to have in-context relevance. To avoid the possibility that toddlers in our study demonstrated context-specific knowledge of novel labels, we did not use the image or the voice of the speakers who provided the labels to test toddlers knowledge and rather had toddlers hear that label spoken by a novel voice.

Second, word learning does not merely entail learning that specific phonetic forms are associated with specific referents. Phonological representations may be manifested by varying phonetic forms with slightly different qualities produced by the same voice or by different voices. Toddlers might be more likely to associate tokens uttered by the same voice with an object, as opposed to making a more general inference that the object has a label that is phonologically invariant (though phonetically variable) across voices. By using an unfamiliar voice during the test phase, we aimed to ensure that toddlers demonstrate generalizable knowledge of novel labels. Experiment 1 was designed to test whether 18-month-olds selectively learn generalizable-across-contexts labels from reliable speakers.
The second primary goal of this study was to investigate the mechanisms behind selective social learning in 18-month-olds. Regarding the mechanisms underlying social learning, one possibility is that toddlers simply recognize the associations between label accuracy and the speaker who provided those labels. They may then extend those associations to the novel labels provided by the same speaker. Recently, Heyes (2017) has suggested that selective social learning is best explained by these kinds of asocial mechanisms. While her arguments were specific to copying behaviors, one could imagine similar arguments extending to word learning. Indeed, Jaswal, Croft, Setia, and Cole (2010; see also Mills, 2013) speculated that children have a "highly robust bias to trust what people-particularly visible speakers-say" (p. 1541). That initial bias might be based on a strong learned association that verbal statements are accurate. The evidence that 2-year-olds do not learn from individuals selectively (Krogh-Jespersen \& Echols, 2012) is consistent with this argument. Similarly, such an approach is consistent with research suggesting that "dumb attentional" associative mechanisms could govern conceptual development and category-based inference in preschoolers (e.g., Sloutsky \& Fisher, 2004; Smith, Jones, \& Landau, 1996).

While associative accounts can be instrumental in explaining early forms of word learning and social inference, it is not clear whether they can account for complex forms of selective social learning. Golinkoff and Hirsh-Pasek (2006), citing empirical evidence from Hollich, Hirsh-Pasek, and Golinkoff (2000), argued that while children's word learning might be governed by associations earlier in development, by 18 months they rely on a combination of cues, including social information, such as referential intent (see also Bloom, 2000). In this case, toddlers should move beyond simple associations among labels, objects, and speakers and incorporate additional information into their inferences concerning whether a novel label for a novel object should be generalized. We considered this in Experiment 2, by replicating the procedure of Experiment 1 with one critical difference. Rather than making assertions about the labels of familiar objects during the familiarization phase of the experiment, the two speakers asked questions about the objects' names. One asked a question about an object using its actual label (e.g., asking "is this a book?" while holding a book); the other used an inappropriate label (e.g., asking "is this a cup?" while holding a ball). By using questions, speakers could present the same accurate or inaccurate label information, while not indicating whether they possessed different knowledge about object labels.

If toddlers are simply recognizing associations among labels, objects, and speakers to make reliability inferences, one might expect similar distinctions between the speakers in Experiments 1 and $2-$ toddlers might believe that when the speaker who used the accurate label in her question makes a statement about the novel label for a novel object, she is more reliable than the speaker who used the inaccurate label in her question. In contrast, if toddlers integrate some understanding of epistemic states into their inferences, 
they should show no difference between the likelihood to generalize either speaker's novel label. Further, because questions have no truth-values to them, toddlers might be likely to consider both speakers equally reliable or equally unreliable as sources of novel information.

\section{2 | EXPERIMENT 1}

Experiment 1 presented an extension of Brooker and PoulinDubois (2013), aimed at determining whether toddlers can use a speaker's past accuracy to guide their generalization of novel labels for novel objects introduced by the speaker. Toddlers first watched a video in which two speakers sat at a table and named different familiar objects. One speaker labeled a set of four objects accurately; the other speaker labeled four different objects inaccurately. Toddlers then saw either the accurate or the inaccurate speaker (between-subject) label two novel objects with novel labels (one at a time).

At test, toddlers saw trials in which a pair of familiar objects or a pair of novel objects were presented on a TV screen. Toddlers heard an audio-recording of an unfamiliar voice utter a label consistent with one of those objects (i.e., "dog" or "ball" if presented with a dog and a ball on the familiar trials and "lif" or "neem" on the novel trials). We recorded toddlers' looking time to the target referents of the labels. We expected that during the test, toddlers would look equally long to the correct objects corresponding to familiar labels, regardless of which speaker labeled the novel objects. However, if toddlers distinguish between the accurate and inaccurate speakers, they should look longer to the correct referents of the novel labels only if the accurate speaker introduced them.

We wish to note two important points about the methodology. First, presenting toddlers with an unfamiliar voice at test critically tests the extent to which they generalize the novel label to that novel object when spoken by different voices. In most experiments examining toddlers' selective word learning (e.g., Brooker \& Poulin-Dubois, 2013; Koenig \& Woodward, 2010, Experiment 1; Krogh-Jespersen \& Echols, 2012) the test utterance is produced by a familiar speaker. Thus, these experiments do not necessarily show that toddlers generalize the meaning of that label to novel contexts (i.e., across speakers). This methodological change also had the benefit of ensuring that test trials were identical for toddlers in all conditions, a control absent from many previous studies.

Second, instead of a forced-choice procedure, toddlers in both experiments were tested in an intermodal preferential looking procedure. This method presented toddlers with a more continuous way to respond to the test question, in which they could weigh responses to both options (as opposed to simply choosing a referent as in previous studies, see Golinkoff, Ma, Song, \& Hirsh-Pasek, 2013). Given that only one study (Brooker \& Poulin-Dubois, 2013) showed selective learning of novel words in 18-month-olds, we wanted to extend these findings using a measure that was potentially more sensitive.

\section{1 | Method}

\subsection{1 | Participants}

Forty 18-month-olds $(M=18.07$ months, $S D=0.43$ months, age range: 16.99-19.02 months; 23 female, 17 male) participated in the study. Participants were recruited from public birth records in an urban area in the Northeastern United States. All were born full-term to monolingual English-speaking families and had no known developmental or hearing disabilities. Families were compensated with a toy, book, or T-shirt at each visit. Additional participants were tested but excluded from the analyses because of hardware malfunction ( $n$ $=2)$, crying or fussiness $(n=6)$, loss of attention to the task $(n=5)$, and impaired vision ( $n=2$ ). We did not collect race, ethnicity, and SES information from participants' parents, but most families were Caucasian and represented middle to upper SES backgrounds.

\subsection{2 | Apparatus}

Toddlers watched visual stimuli presented on a screen (Dell 19" P190S flat screen monitor) mounted on a pegboard approximately 90 $\mathrm{cm}$ in front of the seat. Centered behind the pegboard was a Sentry $110 \mathrm{~A}$ monitor speaker that played the auditory stimuli at a conversational level (75 dB). A video camera (Panasonic WV-BP330 CCTV camera) was situated below the screen, so that the experimenter in the remote control room could record experimental sessions.

\subsection{3 | Materials}

Familiarization and test stimuli included two sets of four familiar objects and two novel objects. One set of four objects included a porcelain cup, a picture book, a stuffed cat, and a rubber ball. The other set of objects included a stuffed dog, a brown shoe, a feeding bottle with a nipple, and a cardboard star painted gold. The objects were chosen to have labels familiar to 18-month-olds, according to the MacArthur CDI lexical norms (Fenson et al., 1993). The labels are listed among 200 most frequently produced and understood words at 18 months in the UCSD Lexical Developmental Norms Database (Dale \& Fenson, 1996). Novel objects were a green drain cover and a yellow tarpaulin clip (Figure 1).

Participants' parents were asked to complete a survey about their child's receptive and productive vocabulary and visual familiarity with object referents of the words used during familiarization. This was included to ensure that participants were familiar with all labels used during the familiarization phase. Parents were also asked to report how many hours their toddlers were exposed to TV and Skype (or similar software) in a typical week. We collected these data to ensure that all participants had had experience with electronic media and that their looking behavior would not be confounded by the novelty of the experience. 


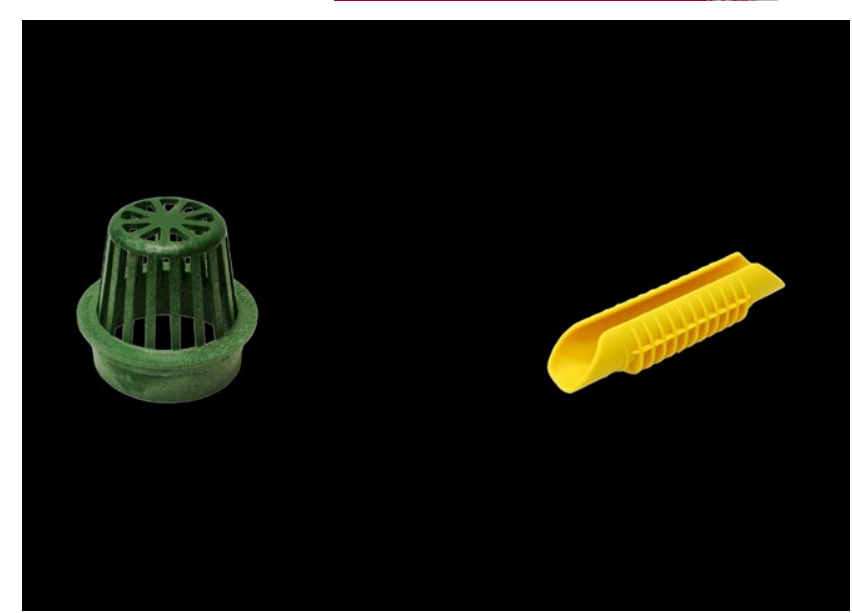

FIGURE 1 Stimuli used during test phase

\subsection{4 | Procedure}

Toddlers were seated in a testing room on the lap of a caregiver who wore active noise reduction headphones playing music to mask the auditory stimuli. The procedure consisted of three phases: familiarization, novel label training, and test (see Table 1). During 3-second intervals between the phases and between different test trials toddlers saw a dark screen. The familiarization phase began with an attention grabber that appeared several times at the areas of the screen where the two speakers appeared later during familiarization.

Toddlers saw an orange circle blinking first on the left and then on the right side of the screen at locations matched with the locations of the faces of the two female informants in the familiarization video. This was done to help coders correctly identify the direction of participants' gaze during the rest of the test trials. Following the attention grabber, participants saw a video, in which the two informants sat at a table with an opaque black cloth on it, in front of an opaque black curtain. Two female speakers were chosen to be similar in appearance to minimize potential artifacts in the looking time data. The speakers were the same race, similar in age, had similar hairstyle and wore similar college insignia sweatshirts of distinct colors (to ensure that toddlers could easily distinguish between them). The speakers took turns bringing out one object, looking at it, and labeling it. One speaker (the accurate speaker) always labeled the objects correctly (e.g., while displaying a star, "This is a star. Star."). The other speaker (the inaccurate speaker), always labeled the objects inaccurately (e.g., while displaying a book, "This is a cat. Cat."). Each speaker labeled a unique set of four objects (eight labeling events in total; see Table 1). This way, only one object was presented at a time, only one speaker labeled it, and each object received only one label. The locations of the object sets, the identity and location of the accurate speaker, and which speaker began showing the objects first were counterbalanced across participants.

After familiarization, the novel label training began. Half of the participants saw the accurate speaker while the other half saw the inaccurate speaker introduce novel labels for two novel objects. Participants were randomly assigned to the accurate or inaccurate speaker condition. During this phase of the procedure, one of the speakers sat at the table. She showed and labeled two novel objects one at a time. The novel labels were nonsense words-"neem" and "lif" (e.g., "Look, a lif! Lif."). We chose the same nonsense CVC words used by Werker, Cohen, Lloyd, Casasola, and Stager (1998) to ensure that the two labels were phonetically distinct yet neither label was more acoustically salient than the other one. This choice of

TABLE 1 Phases of Experiment 1

\begin{tabular}{|c|c|}
\hline Phase: & $\begin{array}{l}\text { Familiarization } \\
\text { ( } 8 \text { labeling events: } \\
4 \text { accurate and } 4 \text { inaccurate) }\end{array}$ \\
\hline Description: & $\begin{array}{l}\text { The accurate speaker and the inaccurate speaker sit at the } \\
\text { same table and take turns to present familiar objects, } 4 \\
\text { objects each. Only one object is present on the table } \\
\text { during a labeling event. Each object receives only one } \\
\text { label. The presenting speaker alternates between looking } \\
\text { at the camera and at the object during a labeling event. } \\
\text { The other speaker looks at the camera with a neutral } \\
\text { expression. }\end{array}$ \\
\hline
\end{tabular}

Novel label training

(4 labeling events:

2 per novel object)

Either the accurate speaker or the inaccurate speaker sits at the table and presents novel objects. The speaker alternates between looking at the camera and at the object she is presenting.

Speaker [presents a novel object 1]: Look, a neem! Neem! Speaker [presents novel object 2]: Look, a lif! Lif!
Test

( 2 novel object trials and 2 familiar object trials)

Two objects (both familiar or both novel) are presented on the screen against a black background. An unfamiliar novel voice asks the participant to look at one of the objects.

Familiar object trial [a toy dog and a ball appear on the screen]: Look, a dog! Where is the dog? Novel object trial [the two novel objects presented earlier appear on the screen]: Look, a lif! Where is the lif? 
labels allowed us to minimize potential confounding effects of label salience. The novel label training was presented twice.

Following the novel label training, toddlers were tested using an Intermodal Preferential Looking Procedure. There were four test trials. In two, participants saw a photograph of a pair of familiar objects (a ball and a dog) on a TV screen. In the other two, they saw a photograph of the pair of novel objects that were labeled during the novel label training. After the photograph was presented for 1 second, participants heard the phrase, "Look, a [label]! Where is the [label]?", with a minimal pause between the two utterances. In the familiar object trials, the label was either "ball" or "dog". One of these labels was uttered by the accurate speaker and the other was uttered by the inaccurate speaker during familiarization. Due to the counterbalancing scheme, $50 \%$ of the time "ball" was uttered by the inaccurate speaker and $50 \%$ of the time "dog" was uttered by the inaccurate speaker.

In the novel object trials, the label was either "lif" or "neem". Utterances in the test trials were provided by a novel voice that did not belong to either the accurate or the inaccurate speaker.

While the novel object trials were the critical experimental trials, the familiar object trials were included as controls to ensure that participants understood the nature of the task, as their knowledge of the meaning of these words should be independent of hearing either the accurate or inaccurate speaker label novel objects (i.e., performance on these trials should indicate mapping of the label to the object in both conditions). Trials were presented in the following order: familiar objects, novel objects, familiar objects, novel objects. The location of the objects was counterbalanced between the trials as well as across participants. During familiarization and novel label training the speakers alternated between looking to the object to cue the object referents of the labels provided and looking to the camera to emulate eye contact with the viewer.

\subsection{5 | Data coding and analysis}

Toddlers' looking behavior was measured during all three phases. The between-subjects independent variable was speaker accuracy during the training phase-whether the accurate or the inaccurate speaker introduced and labeled the novel objects. The dependent measure was the average proportion of looking time to the target object of the total combined looking time to the target and the distractor objects during 3 seconds (30 100-ms bins), starting from 400 ms from the onset of the first mention of the label (to allow toddlers enough time to react to the label heard; see Swingley \& Aslin, 2000). The time interval of 3 seconds was chosen based on overal looking patterns on familiar test trials. On average, toddlers tended to look at the target object the most during the window of analysis and then lost interest and looked at the distractor object or looked away. Figure 2 depicts the proportion of toddlers' looking time at the target from the onset of the first mention of the target word to the end of the window of analysis. All data were coded offline frame by frame ( 1 frame $=33.33 \mathrm{~ms}$ ) using SuperCoder software (Hollich, 2005). Proportions of looking time to the target objects were calculated for 100 -ms bins. A subset of videos (10\%) was coded by a second coder. The intercoder reliability was $97.80 \%$ (Cohen's kappa $=.932$ ) and was calculated based on the exact match of the frames between the coders. For familiarization and test trials, the data from the time intervals during which toddlers did not look at either object were excluded from analyses.

\section{2 | Results}

Based on parental report, participants knew on average 6.73 of 8 words used in familiarization. Thirty-seven out of 40 toddlers knew both familiar words-dog and ball-that were used during the test. The remaining three toddlers knew one of the words.

We analyzed the effects of counterbalancing factors, toddlers' verbal competence, media exposure reported by parents, and gender to ensure that individual differences between participants and biases potentially arising from the experimental design did not explain the observed differences in toddlers' looking behavior across the conditions. Mann-Whitney tests showed no significant effects, all $Z$-scores $\leq 1.58$, all $p$-values $\geq .18$. These variables were not considered in any further analyses.

Looking behavior during the familiarization and novel label training phases was analyzed separately to ensure that toddlers were equally attentive when both accurate and inaccurate speakers presented the objects (see Table 2). For familiarization trials,

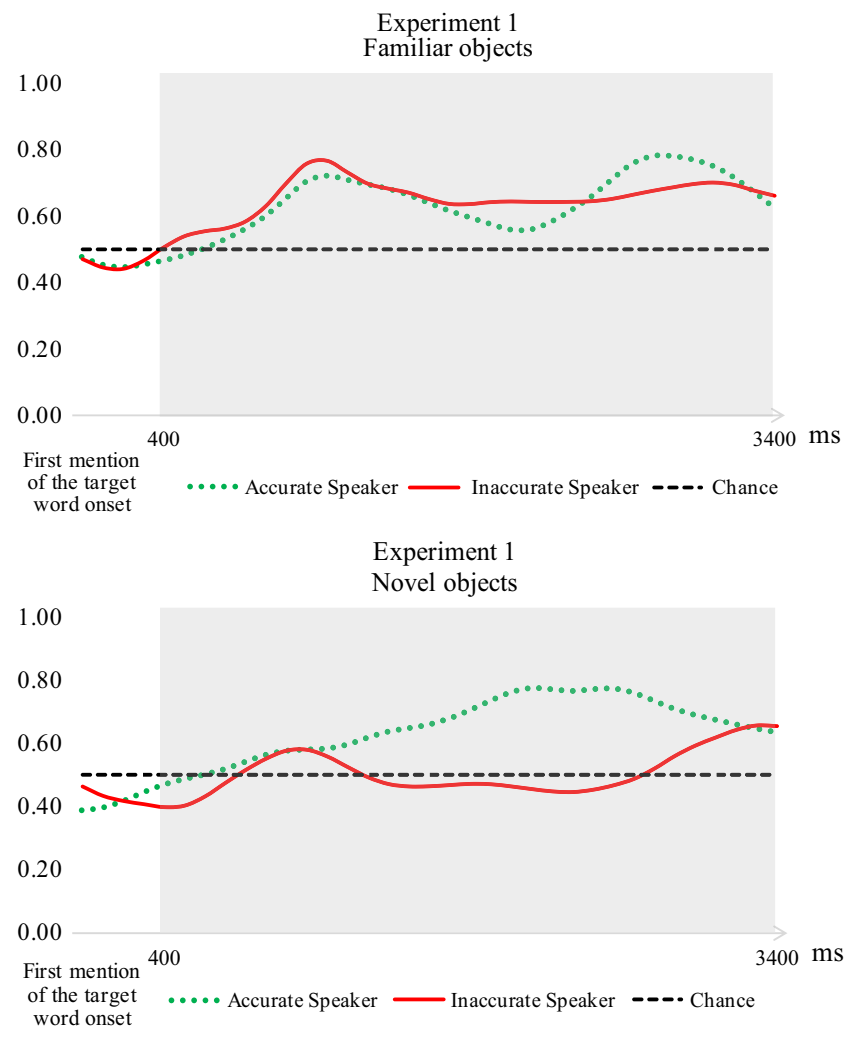

FIGURE 2 Experiment 1: Proportion of looking time to the target object (smoothed) from the onset of the target word. The window of analysis extended from $400 \mathrm{~ms}$ to $3400 \mathrm{~ms}$ post onset 


\begin{tabular}{|c|c|c|c|c|}
\hline & & $\begin{array}{l}\text { Accurate } \\
\text { Speaker } \\
\text { Condition }\end{array}$ & $\begin{array}{l}\text { Inaccurate Speaker } \\
\text { Condition }\end{array}$ & \\
\hline & Phase & $M(S D)$ & $M(S D)$ & $p$-value \\
\hline \multirow[t]{6}{*}{ Experiment 1} & $\begin{array}{l}\text { Familiarization - } \\
\text { Inaccurate Speaker }\end{array}$ & $.96(.04)$ & $.96(.05)$ & .55 \\
\hline & $\begin{array}{l}\text { Familiarization - Accurate } \\
\text { Speaker }\end{array}$ & $.98(.03)$ & $.96(.05)$ & .31 \\
\hline & Label 1 ("Neem") Training & $.99(.03)$ & $.98(.05)$ & .69 \\
\hline & Label 2 ("Lif") Training & $.99(.05)$ & $.96(.08)$ & .95 \\
\hline & Test - Familiar objects & $.63(.11)$ & $.63(.11)$ & .90 \\
\hline & Test - Novel objects & $.63(.13)$ & $.47(.18)$ & .007 \\
\hline \multirow[t]{6}{*}{ Experiment 2} & $\begin{array}{l}\text { Familiarization - } \\
\text { Inaccurate Speaker }\end{array}$ & $.97(.04)$ & $.96(.05)$ & .17 \\
\hline & $\begin{array}{l}\text { Familiarization - Accurate } \\
\text { Speaker }\end{array}$ & $.98(.03)$ & $.96(.05)$ & .37 \\
\hline & Label 1 ("Neem") Training & $.99(.03)$ & $.98(.05)$ & .70 \\
\hline & Label 2 ("Lif") Training & $.99(.05)$ & $.97(.08)$ & .95 \\
\hline & Test - Familiar objects & $.65(.13)$ & $.69(.15)$ & .12 \\
\hline & Test - Novel objects & $.49(.19)$ & $.39(.19)$ & .55 \\
\hline
\end{tabular}

TABLE 2 Proportion of looking time to speakers during familiarization and novel label training the numbers in Table 2 represent proportions of looking time at the target object and/or the presenting speaker of the total looking time at the screen, excluding looking away. Because there were no stimuli that would distract the child from the target object and the speaker during the novel label training trials, the numbers represent the proportions of looking time at the target object and/ or the presenting speaker of the total duration of the trial. MannWhitney tests showed no significant differences in looking time during familiarization or novel label training phases, all Z-scores $\leq 1.08$, all $p$-values $\geq .31$. Moreover, inspection of Table 2 shows that the majority of the time, toddlers attended to the presenting speaker.

We conducted an omnibus ANOVA with the proportion of looking time to the target object as a dependent measure, condition (accurate vs. inaccurate speaker during the novel label training) as a between-subjects factor, and trial type (novel vs. familiar objects) as a within-subject factor. There was a significant effect of trial type, $F(1,38)=5.68, p=.022, \eta_{p}{ }^{2}=.13$, with toddlers looking longer on average to familiar $(M=63.53 \%)$ than to novel $(M=56.24 \%)$ objects. There was also a significant effect of condition, $F(1,38)=4.66, p$ $=.037, \eta_{p}{ }^{2}=.11$, with toddlers looking longer to the target objects in the accurate speaker condition ( $M=63.35 \%)$ than in the inaccurate speaker condition $(M=56.41 \%)$, and a significant interaction between condition and trial type, $F(1,38)=5.87, p=.02, \eta_{p}{ }^{2}=.13$.

Independent sample two-tailed $t$ tests were used to analyze differences in the proportions of looking time to the novel and familiar target objects across conditions. As Figure 3 illustrates, toddlers in the accurate speaker condition looked to the novel target labels significantly more than toddlers in the inaccurate speaker condition, $t(38)=-2.85, p=.007, d=0.90$ (accurate speaker $M=63.41 \%, S D=$
13.71; inaccurate speaker $M=47.07 \%, S D=17.86)$. There was no significant difference in looking to the familiar target objects between the accurate and inaccurate conditions, $M=63.29 \%, S D=11.67$ vs. $M=63.76 \%, S D=11.26, t(38)=.127, p=.90$.

We further examined the proportion of looking time to the target objects with respect to chance (50\%) using two-tailed one-sample $t$ tests. On the familiar object trials, toddlers looked significantly above chance in both accurate and inaccurate speaker conditions, $t(19)=4.81, p=.000 ; d=1.14$ and $t(19)=5.46, p=.000 ; d=1.22$. In contrast, on the novel object trials, only toddlers in the accurate speaker condition looked significantly above chance, $t(19)=4.37, p=$ $.000 ; d=0.97$. Toddlers in the inaccurate speaker condition did not look at the target objects significantly above chance, $t(19)=-0.23$, $p=.82$.

\section{3 | Discussion}

Using a continuous measure-toddlers' looking time in an IPLP procedure-we showed that 18-month-olds were more likely to map novel labels to novel objects and generalize them to a novel context when they learned those labels from informants who previously labeled familiar objects accurately rather than inaccurately. Our results extend the findings by Brooker and Poulin-Dubois (2013), who showed that toddlers responded differently to a disambiguation paradigm when labels were presented by accurate versus inaccurate speakers. Critically, toddlers made a similar inference here when they heard an unfamiliar voice during the test trial. This ensured that all toddlers were exposed to exactly the same stimuli during test trials and that they were generalizing newly learned labels to novel social conditions. 


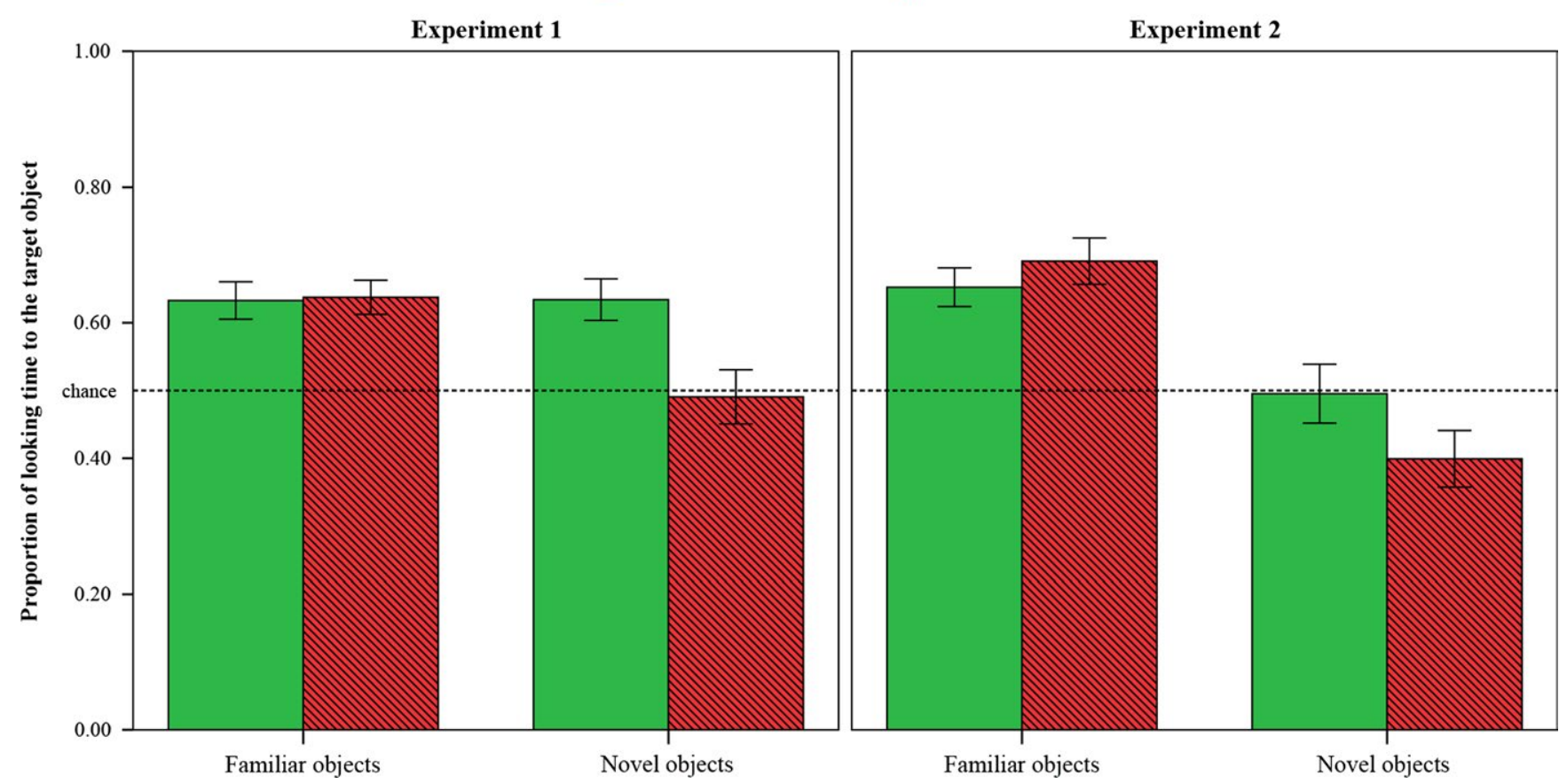

FIGURE 3 Proportion of looking time to the target object during $3 \mathrm{~s}$, starting from $400 \mathrm{~ms}$ after the first mention of the target label

Importantly, during familiarization the two speakers were presented on the screen side by side. Vanderbilt, Heyman, and Liu (2014) examined how such conflicting information affected 3- and 4-year-olds' use of speaker accuracy. They showed that if a conflict was presented at test (the accurate and inaccurate speakers provided different novel labels for the same novel object), preschoolers used speakers' accuracy. Without such a conflict (i.e., if speakers only conflicted during familiarization), preschoolers trusted an accurate and an inaccurate speakers' subsequent novel labels equally. In the present study, the speakers never contrasted labels for objects at either stage of the procedure, but there was a contrast between an accurate and inaccurate speaker during familiarization before the child heard novel labels for novel objects from only one speaker. Experiment 1 suggests that this subtler contrast-simply seeing accurate and inaccurate informants together, even if they never provide conflicting information-was sufficient for our younger children (and possibly the older children investigated by Vanderbilt et al., 2014) to trust the informants selectively.

The results of Experiment 1 provide evidence that toddlers possess the ability to track informants' accuracy and use such information for subsequent inferences about the meaning of novel labels. This account is consistent with various findings demonstrating infants' selective trust of informants in domains other than word learning (e.g., Poulin-Dubois, Brooker, \& Polonia, 2011; Stenberg, 2013; Tummeltshammer et al., 2014; Zmyj et al., 2010). However, these results leave open the question about the mechanisms underlying toddlers' reliability judgments. One possibility is that toddlers generalize the association between speaker's identity and the accuracy of the labels she provides for familiar objects to all new information provided by the same speaker. This mechanism does not involve judgments about speakers' epistemic states and is based on superficial associative information. Alternatively, toddlers might use speaker's utterances to make inferences regarding her knowledge of object names and, hence, her competence in naming. Southgate, Chevallier, and Csibra (2010) have demonstrated that at 17 months toddlers are already sensitive to informants' epistemic states and rely on that information in novel object naming contexts. It is possible that toddlers also incorporate inferences about epistemic states into their reliability judgments. Instead of simply relying on associative information, such information might be integrated with judgments of epistemic knowledge.

To investigate the mechanisms underlying toddlers' reliability judgments, in Experiment 2, we replaced statements about object names during familiarization with questions mentioning the same labels. Infants as young as 15 months old understand subject questions (Seidl, Hollich, \& Jusczyk, 2003) and infants as young as 7 months are sensitive to prosodic cues that indicate the difference between questions and statements (Soderstrom, Ko, \& Nevzorova, 2011). Liszkowski, Carpenter, and Tomasello (2008) showed that 12-month-olds understood the pragmatics of questions based on the speaker's access to information and responded to her questions appropriately. Shatz (1978) similarly showed that toddlers can comprehend direct requests for information (e.g., "Is this an apple?") as well as indirect requests for actions ("Can you jump?"); toddlers respond in an appropriate manner to both types of questions. These findings suggest that young children have an ability to appreciate pragmatic contexts of questions, make inferences about askers' intentions, and generate responses accordingly. In Experiment 2, the 
speakers were instructed to emulate genuine questions, with facial expressions and intonations signaling a request for information rather than rhetorical questions.

Such a method potentially allows us to distinguish between these two mechanisms for selective learning. If toddlers judge speaker accuracy on the basis of whether they hear a speaker provide appropriate label-object pairs, then one would expect similar responses here as in Experiment 1. In contrast, if toddlers understand that questions, unlike statements, lack truth-values, then questions will indicate the same level of accuracy regardless of whether they contain the appropriate or inappropriate label-object pairings.

\section{EXPERIMENT 2}

In Experiment 2, we consider whether toddlers are attending to speakers' epistemic knowledge as opposed to a simple label-objectspeaker association. We replicated the procedure of Experiment 1 with one modification: during familiarization, the two speakers asked questions about labels of objects familiar to toddlers instead of making statements about the objects' labels; all other aspects of the procedure were the same.

\section{1 | Method}

\subsection{1 | Participants}

Forty 18 -month-olds $(M=17.67$ months, $S D=0.54$ months, age range: $16.82-18.83$ months; 14 female, 26 male) participated in the study. Recruitment procedures, eligibility requirements and compensation were the same as in Experiment 1. Additional participants were tested but excluded from the analyses because of crying or fussiness $(n=1)$ and a substantial delay in motor development ( $n$ =1). We did not collect race, ethnicity, and SES information from participants' parents. Most families represented middle to upper SES and were Caucasian.

\subsection{2 | Apparatus}

The same apparatus was used as in Experiment 1.

\subsection{3 | Materials}

All materials were the same as in Experiment 1.

\subsection{4 | Procedure}

The same procedure as in Experiment 1 was employed, except that during familiarization the two speakers (the same speakers as in Experiment 1; the speakers were blind to the experimental hypothesis) asked questions instead of making statements about the labels of familiar objects (e.g., "Hmm... Is this a star? A star?"). During novel label training, the speakers made statements about the labels of the novel objects, as in Experiment 1. To keep terminology consistent across the two experiments, we refer to the speaker who mentioned correct labels of the objects in her questions as the accurate speaker and the speaker who mentioned incorrect labels of objects in her questions as the inaccurate speaker. Stimuli were edited to ensure that the length of utterances and volume levels matched Experiment 1.

\subsection{5 | Data coding and analysis}

The same coding and analysis procedures were used as in Experiment 1. Figure 4 depicts the proportion of toddlers' looking time at the target objects from the onset of the first mention of the target word to the end of the window of analysis.

\section{2 | Results and discussion}

On average, toddlers knew 6.50 of 8 words used during familiarization, based on parental report. Thirty-eight out of 40 toddlers knew both familiar words-dog and ball-that were used during the test. The remaining two toddlers knew one of the words.

The effects of counterbalancing factors, children's gender, children's verbal competence, and media exposure reported by parents did not explain observed differences in toddlers' looking behavior across conditions; Mann-Whitney tests showed no significant effects, all $Z$-scores $\leq 1.69$, all $p$-values $\geq .095$. As in Experiment 1 ,
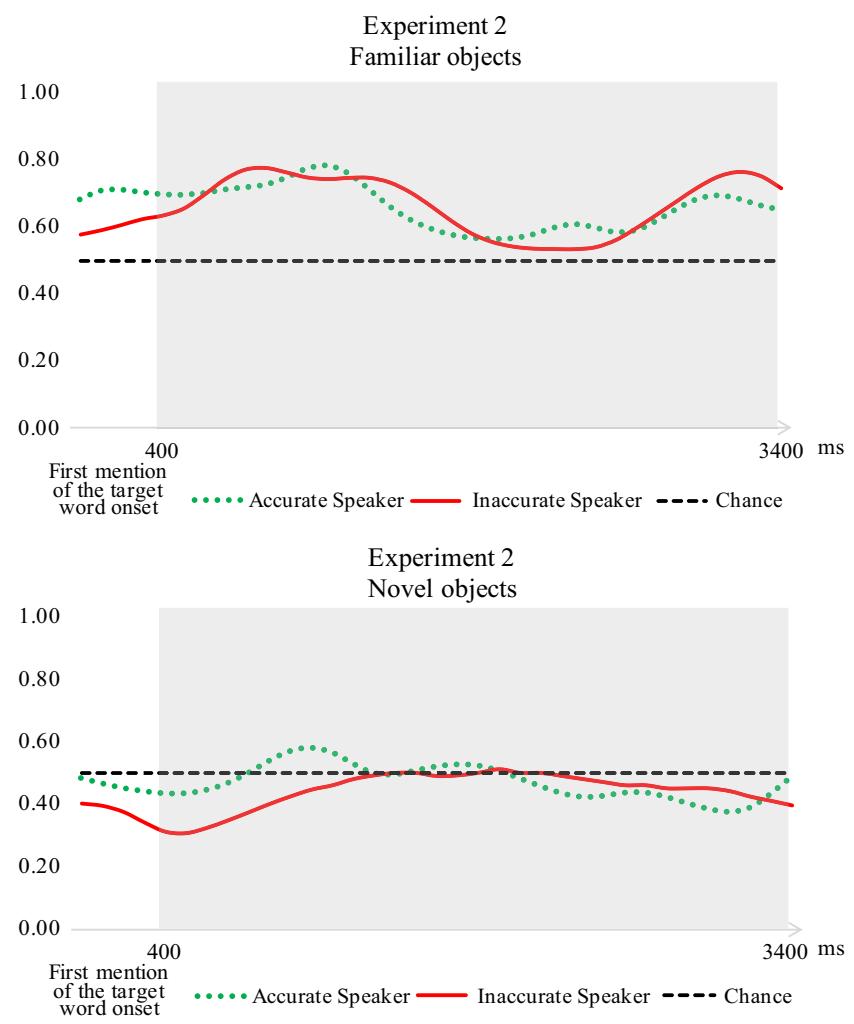

FIGURE 4 Experiment 2: Proportion of looking time to the target object (smoothed) from the onset of the target word. The window of analysis extended from $400 \mathrm{~ms}$ to $3400 \mathrm{~ms}$ post onset 
Mann-Whitney tests also showed no significant differences between the conditions in looking time during familiarization and novel label training, all Z-scores $\leq 1.39$, all $p$-values $\geq .17$; see Table 2 . These variables were not considered in further analyses.

We conducted an omnibus ANOVA with the proportion of looking time to the target object as a dependent measure and with trial type (novel vs. familiar objects) as a within-subject factor and condition (accurate vs. inaccurate speaker during the novel label training) as a between-subjects factor. The analyses revealed a significant effect of trial type, $F(1,38)=28.72, p=.00, \eta_{p}{ }^{2}=.43$, with toddlers performing better on familiar object trials $(M=67.18)$ than on novel object trials ( $M=44.73$ ). In contrast to Experiment 1 , there were no significant effects of condition, $F(1,38)=0.79 p=.38$. The trial type by condition interaction was also not significant, $F(1,38)=2.59, p=$ $.116, \eta_{\mathrm{p}}^{2}=.06$.

In contrast to Experiment 1, the results of planned comparison between the accurate and inaccurate speakers on the novel trials showed no significant difference between the conditions for the novel objects (see Figure 3), $t(38)=-1.59, p=.55$ (accurate speaker $M=49.54 \%, S D=19.44$; inaccurate speaker $M=39.92 \%, S D=18.67$ ). There was also not a significant difference in toddlers' looking to the familiar target objects, $t(38)=86.5, p=.121$ (accurate speaker $M=$ $65.25 \%, S D=12.75$; inaccurate speaker $M=69.10 \%, S D=15.29$ ).

We again compared the proportion of looking time in each condition to chance, using one-sample $t$ tests. On familiar object trials, toddlers looked significantly above chance in both conditions, $t(19)$ $=5.35, p<.01, d=1.20$ and $t(19)=5.59, p<.01, d=1.25$, for the accurate and inaccurate speaker conditions, respectively. On novel label trials, toddlers in the accurate speaker condition looked to the target objects at chance, $t(19)=-.106, p=.92$. In the inaccurate speaker condition, the proportion of toddlers' looking time to the novel target objects was below chance, $t(19)=-2.41, p=.026$. The below-chance looking time may be an artifact, due to toddlers in this condition looking at the distractor object while the target word was being pronounced and immediately afterwards. ${ }^{2}$

These results suggest that toddlers are not relying only on associations between correct (or incorrect) word-label pairs and speaker identity in learning and generalizing new words. Moreover, toddlers appear to treat questions and statements made by individuals during familiarization differently. Unlike statements, which toddlers seem to believe indicate speakers' knowledge of novel object labels, questions do not afford information for the generalization of those labels. Indeed, while toddlers continued to demonstrate knowledge of the labels for familiar objects, they seemed to learn little from either speaker's utterances in Experiment 2. Because questions lack truth-values, toddlers did not think that either speaker was a better source of word knowledge than the other one. However, it is possible that because the speakers were emulating genuine request for information during familiarization, toddlers interpreted the pragmatics of the situation to signal that neither speaker was knowledgeable enough. Perhaps 18-month-olds judged that speakers who are uncertain about the meanings of labels so familiar that even toddlers know them are too ignorant to trust for new labels. The results of
Experiment 2, therefore, suggest that toddlers do not simply extrapolate their associations between speaker identity and her accuracy in naming to the novel information she generates in the future. Instead, they incorporate the judgments about speakers' epistemic competence in selective generalization of novel labels.

\section{4 | GENERAL DISCUSSION}

In the present study we pursued two major goals: to replicate and extend earlier findings suggesting that 18-month-olds selectively trust reliable speakers, and to shed light on the mechanisms that might underlie their reliability judgments. In Experiment 1, we presented 18-month-olds with two speakers. One always labeled a set of familiar objects accurately; the other labeled a set of different familiar objects inaccurately. Children then saw either the accurate or the inaccurate speaker introduce novel labels for novel objects (between subject), and were tested for their generalization of that label-object relation in an intermodal preferential looking procedure. Eighteen-month-olds in both conditions recognized familiar label-familiar object relations, but only recognized novel label-novel object relations when those labels were introduced by the previously accurate speaker.

This finding corroborates and extends work by Brooker and Poulin-Dubois (2013) and shows that 18-month-olds selectively generalize novel word-object pairs to novel contexts when the words were presented by accurate speakers. This finding is also consistent with results suggesting that by the second half of the first year, infants generalize object labels across contexts (e.g., Henderson \& Graham, 2005; Graham, Stock, \& Henderson, 2006; Henderson \& Woodward, 2012; Novack, Henderson, \& Woodward, 2014). More generally, these data are consistent with the hypothesis that young children are sensitive to social cues that signal communicative intent, and that such cues might guide learning behavior (e.g., Csibra, 2010; Csibra \& Gergeley, 2009). Ostensive communicative signals, such as eye contact, may create referential expectations and the assumption of generalizability of new information in a child's mind. Intonation, social referencing, affect, and body movements can all be used as indicators of pretend play (e.g., Lillard \& Witherington, 2004), to communicate meaning (e.g., Fernald, Taeschner, Dunn, Papousek, de Boysson-Bardies, \& Fukui, 1989), or to indicate intentions (e.g., Baldwin, 1993; Csibra \& Gergeley, 2009). If toddlers are sensitive to social context in which they learn new words, it is possible that they would incorporate this information into their selective inferences for word learning.

The purpose of Experiment 2 was to explore the mechanisms that might underlie this early ability. By using questions instead of statements during familiarization, we retained the label-objectspeaker association inherent in Experiment 1, but eliminated the cues that helped signal the difference in speakers' epistemic states. Demonstrating that toddlers did not generalize the labels from either speaker to novel contexts suggests that they are not just using the label-object-speaker associations but rather interpreting statements 
as reflecting speakers' epistemic competence. Integrating the discerning responses of toddlers between the conditions in Experiment 1 with the chance-level responding in Experiment 2,18-month-olds appear capable of tracking that epistemic competence and using it to generalize information from different sources judiciously.

One might be concerned whether toddlers found it believable that adult speakers do not know the labels of familiar objects and have to request that information in Experiment 2. However, the results of Experiment 1 speak against this interpretation. If toddlers found it hard to believe that adults did not know the names of familiar objects, they would have discounted the inaccurate speaker's statements as not being reflexive of her epistemic knowledge; given that this was not the case in Experiment 1, there is reason to believe that in Experiment 2, children believed that the speakers were requesting information about the object names.

Another concern is that toddlers in Experiment 2 treated the questions about object names as rhetorical. Two pieces of evidence speak against this possibility. First, if toddlers in Experiment 2 truly treated questions about object names as rhetorical, they should have trusted the accurate speaker and performed above chance on the test trials (i.e., similar to performance in Experiment 1). Second, Shatz (1978) showed that even toddlers with lower verbal competence (MLU <3 words) responded in an appropriate manner to information requests similar to ones we used (e.g., "Is this an apple?") $81 \%$ of the time. This suggests that the children in Experiment 2 would be more likely to interpret these utterances as information seeking. It is worth noting that toddlers treated both speakers as unreliable in Experiment 2. Such a finding potentially speaks against the possibility that children have a default bias to trust others' information. Alternatively, such a bias might be based on the fact that typically we interact with accurate informants, making such a bias a learned response (Jaswal et al., 2010). If so, it is possible that 18-month-olds have not learned enough about exchanging knowledge through verbal communication. Eighteenmonth-olds might be early enough in the process of learning to exchange information that they have no default assumptions about others. Of course, between 18 and 24 months, toddlers also develop higher sensitivity to confidence cues, which they rely on in deciding whom to trust (Brosseau-Liard \& Poulin-Dubois, 2014). It is possible that children see speakers who ask questions as being less confident than speakers who make statements, making them both unreliable. This interpretation is consistent with evidence reported by Zmyj et al. (2010) who showed that 14-month-olds rely on a combination of competence and confidence cues to decide which model's actions to imitate. It would be interesting to track how older children treat speakers who only ask questions. One could argue that by the time children are preschoolers, they might have learned that the majority of utterances are truthful without evidence to the contrary, making both of the question-askers in Experiment 2 reliable sources of novel information; alternatively, one could argue that such questions reveal ignorance on the part of the informants, making both question-askers unreliable. This is an open empirical question.
We conclude by suggesting that by 18 months, children use social knowledge, based on pragmatic differences between statements and questions, to make judgments about the epistemic knowledge of others' testimony for word learning. Our findings signal a more general capacity to integrate social and statistical information into reliability judgments, which develops as toddlers reach better understanding of social and pedagogical cues (see Sobel \& Kushnir, 2013). While specific social cues that toddlers may use to make reliability judgments remain subject to further investigation, the present study showed that 18-month-olds generalize new words learned from accurate speakers and extended these results by demonstrating that sensitivity to more sophisticated social cues seem best able to explain the mechanism by which children engage in such inferences.

\section{ACKNOWLEDGEMENTS}

This research was funded by the National Science Foundation grant (1223777) to DMS and National Institutes of Health grant (HD068501) to JLM. We would like to thank Anneke Elmhirst, Emily Davis, and Lori Rolfe for assistance with stimuli design, data collection and coding.

\section{ENDNOTES}

${ }^{1}$ One exception is Koenig and Woodward (2010): in Experiments 2 and 3, toddlers' knowledge was tested by the source of novel information and by an unfamiliar speaker.

${ }^{2}$ Toddlers' looking time to the target objects from $800 \mathrm{~ms}$ to $3400 \mathrm{~ms}$ after the target word onset was not significantly different from chance, $t(19)=$ $-2.11, p=.05$

\section{REFERENCES}

Baldwin, D.A. (1993). Infants' ability to consult the speaker for clues to word reference. Journal of Child Language, 20, 395-418.

Bloom, P. (2000). How children learn the meanings of words. Cambridge, MA: The MIT Press.

Brooker, I., \& Poulin-Dubois, D. (2013). Is a bird an apple? The effect of speaker labeling accuracy on infants' word learning, imitation, and helping behaviors. Infancy, 18, E46-E68.

Brosseau-Liard, P.E., \& Poulin-Dubois, D. (2014). Sensitivity to confidence cues increases during the second year of life. Infancy, 19, 461-475.

Clément, F., Koenig, M., \& Harris, P. (2004). The ontogenesis of trust. Mind \& Language, 19, 360-379.

Csibra, G. (2010). Recognizing communicative intentions in infancy. Mind \& Language, 25, 141-168.

Csibra, G., \& Gergely, G. (2009). Natural pedagogy. Trends in Cognitive Sciences, 13, 148-153.

Dale, P.S., \& Fenson, L. (1996). Lexical development norms for young children. Behavioral Research Methods, Instruments, \& Computers, 28, 125-127.

Fenson, L., Dale, P.S., Reznick, J.S., Thal, D., Bates, E., Hartung, J.P., ... Reilly, J.S. (1993). The MacArthur communicative development inventories. User's guide and technical manual. San Diego, CA: Singular.

Fernald, A., Taeschner, T., Dunn, J., Papousek, M., de Boysson-Bardies, B., \& Fukui, I. (1989). A cross-language study of prosodic modifications 
in mothers' and fathers' speech to preverbal infants. Journal of Child Language, 16, 477-501.

Golinkoff, R.M., \& Hirsh-Pasek, K. (2006). Baby wordsmith: From associationist to social sophisticate. Current Directions in Psychological Science, 15, 30-33.

Golinkoff, R.M., Ma, W., Song, L., \& Hirsh-Pasek, K. (2013). Twentyfive years using the intermodal preferential looking paradigm to study language acquisition: What have we learned? Perspectives on Psychological Science, 8, 316-339.

Graham, S.A., Stock, H., \& Henderson, A.M. (2006). Nineteen-montholds' understanding of the conventionality of object labels versus desires. Infancy, 9, 341-350.

Henderson, A.M., \& Graham, S.A. (2005). Two-year-olds' appreciation of the shared nature of novel object labels. Journal of Cognition and Development, 6, 381-402.

Henderson, A.M., Graham, S.A., \& Schell, V. (2015). 24-month-olds' selective learning is not an all-or-none phenomenon. PLoS ONE, 10, e0131215.

Henderson, A.M., \& Woodward, A.L. (2012). Nine-month-old infants generalize object labels, but not object preferences across individuals. Developmental Science, 15, 641-652.

Hendrickson, K., \& Sundara, M. (2017). Fourteen-month-olds' decontextualized understanding of words for absent objects. Journal of Child Language, 44, 239-254.

Heyes, C. (2017). When does social learning become cultural learning? Developmental Science, 20, e12350.

Hollich, G. (2005). Supercoder: A program for coding preferential looking (Version 1.5). Computer Software]. West Lafayette, IN: Purdue University.

Hollich, G., Hirsh-Pasek, K., \& Golinkoff, R.M. (2000). II. The emergentist coalition model. Monographs of the Society for Research in Child Development, 65, 17-29.

Jaswal, V.K., Croft, A.C., Setia, A.R., \& Cole, C.A. (2010). Young children have a specific, highly robust bias to trust testimony. Psychological Science, 21, 1541-1547.

Koenig, M.A., Clément, F., \& Harris, P.L. (2004). Trust in testimony: Children's use of true and false statements. Psychological Science, 15, 694-698.

Koenig, M.A., \& Harris, P.L. (2005). Preschoolers mistrust ignorant and inaccurate speakers. Child Development, 76, 1261-1277.

Koenig, M.A., \& Woodward, A.L. (2010). Sensitivity of 24-month-olds to the prior inaccuracy of the source: Possible mechanisms. Developmental Psychology, 46, 815-826.

Krogh-Jespersen, S., \& Echols, C.H. (2012). The influence of speaker reliability on first versus second label learning. Child Development, 83, 581-590.

Lillard, A.S., \& Witherington, D.C. (2004). Mothers' behavior modifications during pretense and their possible signal value for toddlers. Developmental Psychology, 40, 95-113.

Liszkowski, U., Carpenter, M., \& Tomasello, M. (2008). Twelve-montholds communicate helpfully and appropriately for knowledgeable and ignorant partners. Cognition, 108, 732-739.
Mills, C.M. (2013). Knowing when to doubt: Developing a critical stance when learning from others. Developmental Psychology, 49, 404-418.

Novack, M.A., Henderson, A.M., \& Woodward, A.L. (2014). Twelvemonth-old infants generalize novel signed labels, but not preferences across individuals. Journal of Cognition and Development, 15, 539-550.

Poulin-Dubois, D., Brooker, I., \& Polonia, A. (2011). Infants prefer to imitate a reliable person. Infant Behavior and Development, 34, 303-309.

Saussure, F.D. (1966). Course in general linguistics (edited by Charles Bally and Albert Sechehaye, translated by Wade Baskin). New York: McGraw-Hill.

Seidl, A., Hollich, G., \& Jusczyk, P.W. (2003). Early understanding of subject and object wh-questions. Infancy, 4, 423-436.

Shatz, M. (1978). On the development of communicative understandings: An early strategy for interpreting and responding to messages. Cognitive Psychology, 10, 271-301.

Sloutsky, V.M., \& Fisher, A.V. (2004). Induction and categorization in young children: A similarity-based model. Journal of Experimental Psychology: General, 133, 166-188.

Smith, L.B., Jones, S.S., \& Landau, B. (1996). Naming in young children: A dumb attentional mechanism? Cognition, 60, 143-171.

Sobel, D.M., \& Kushnir, T. (2013). Knowledge matters: How children evaluate the reliability of testimony as a process of rational inference. Psychological Review, 120, 779-797.

Soderstrom, M., Ko, E.S., \& Nevzorova, U. (2011). It's a question? Infants attend differently to yes/no questions and declaratives. Infant Behavior and Development, 34, 107-110.

Southgate, V., Chevallier, C., \& Csibra, G. (2010). Seventeen-month-olds appeal to false beliefs to interpret others' referential communication. Developmental Science, 13, 907-912.

Stenberg, G. (2013). Do 12-month-old infants trust a competent adult? Infancy, 18, 873-904.

Swingley, D., \& Aslin, R.N. (2000). Spoken word recognition and lexical representation in very young children. Cognition, 76, 147-166.

Tummeltshammer, K.S., Wu, R., Sobel, D.M., \& Kirkham, N.Z. (2014). Infants track the reliability of potential informants. Psychological Science, 25, 1730-1738.

Vanderbilt, K.E., Heyman, G.D., \& Liu, D. (2014). In the absence of conflicting testimony young children trust inaccurate informants. Developmental Science, 17, 443-451.

Werker, J.F., Cohen, L.B., Lloyd, V.L., Casasola, M., \& Stager, C.L. (1998). Acquisition of word-object associations by 14 -month-old infants. Developmental Psychology, 34, 1289-1309.

Zmyj, N., Buttelmann, D., Carpenter, M., \& Daum, M.M. (2010). The reliability of a model influences 14-month-olds' imitation. Journal of Experimental Child Psychology, 106, 208-220.

How to cite this article: Luchkina E, Sobel DM, Morgan JL. Eighteen-month-olds selectively generalize words from accurate speakers to novel contexts. Dev Sci. 2018;e12663. https://doi.org/10.1111/desc.12663 